\title{
The antagonistic effects and mechanisms of microRNA-26a action in hypertensive vascular remodeling
}

\author{
Wenqian Zhang ${ }^{1}$, Qiaozhu Wang ${ }^{1}$, Xin Xing ${ }^{1}$, Lijun Yang ${ }^{1}$, Min Xu ${ }^{1}$, Chunhui Cao ${ }^{1}$, Rong \\ Wang ${ }^{1}$, Weicheng $\mathrm{Li}^{1}$, Xiaolin $\mathrm{Niu}^{1}$, and Dengfeng $\mathrm{GaO}^{2}$ \\ ${ }^{1}$ Xi'an Jiaotong University Second Affiliated Hospital \\ ${ }^{2} 2$ nd affiliated hospital of Xi'an Jiaotong University
}

July 30, 2020

\begin{abstract}
Background and Purpose: Hypertensive vascular remodeling (VR) is responsible for end-organ damage and is the result of increased extracellular matrix accumulation and excessive vascular smooth muscle cell (VSMC) proliferation. MicroRNA-26a (miR-26a), a non-coding small RNA, is involved in multiple cardiovascular diseases. We aimed to validate the effect and mechanisms of miR-26a in hypertensive VR. Experimental Approach: Spontaneously hypertensive rats (SHRs) were injected intravenously with recombinant adeno-associated virus-miR-26a. In vitro experiments, angiotensin II (AngII)-induced VSMCs were transfected with miR-26a mimic or inhibitor. Key Results: We found miR-26a downregulated in the thoracic aorta and plasma of SHRs. Overexpression of miR-26a inhibited extracellular matrix deposition by targeting connective tissue growth factor (CTGF) and mitigated VSMC proliferation by regulating the enhancer of zeste homolog 2 (EZH2)/p21 pathway both in vitro and in vivo. AngII-mediated Smad3 activation suppressed miR-26a expression, which in turn promoted Smad3 activation via targeted regulation of Smad4, leading to further downregulation of miR-26a. Conclusion and Implications: Our study reveals that AngII stimulates a Smads/miR-26a positive feedback loop, which further reduces miR-26a expression, leading to collagen production and VSMC proliferation and consequently, VR. MiR-26a has an antagonistic effect on hypertensive VR and can be a strategy for treating hypertensive VR.
\end{abstract}

\section{Abbreviations}

AngII: angiotensin II

Col: collagen

CTGF: connective tissue growth factor

CVF: collagen volume fraction

ECM: extracellular matrix

EZH2: enhancer of zeste homolog 2

GAPDH: glyceraldehyde-3-phosphate dehydrogenase

$\mathrm{H} \& \mathrm{E}$ : hematoxylin-eosin

LD: lumen diameter

miR: microRNA

MT: media thickness 
MuT: mutant-type

OD: optical density

PBS: phosphate buffered saline

PCNA: proliferating cell nuclear antigen

p-Smad3: phospho-Smad3

qRT-PCR: quantitative real-time PCR

rAAV: recombinant adeno-associated virus

SBP: systolic blood pressure

SHR: spontaneously hypertensive rat

siRNA: small interfering RNA

VR: vascular remodeling

VSMC: vascular smooth muscle cell

WKY: Wistar Kyoto rat

\section{Introduction}

Vascular remodeling (VR) persists throughout the course of hypertension and is characterized by chronic, continuous, and complex changes in vascular structure and abnormalities in vascular functions (Hayashiet al. , 2009; Lemarie et al. , 2010; Zhang et al. , 2019). VR, as an important indicator of the progression of hypertension, is closely related to the severity and prognosis of the disease (Mulvany, 2012; Magnussen, 2017; Brown et al. , 2018). Angiotensin II (AngII)-induced dysfunction of vascular smooth muscle cells (VSMCs) plays a critical role in hypertensive VR (Das et al. , 2018), but the potential molecular mechanisms remain poorly understood. Therefore, study of new therapeutic targets for hypertensive VR and exploring potential molecular mechanisms are of benefit.

MicroRNAs (miRs) are highly conserved, small non-coding RNAs that modulate the function of VSMCs, such as their proliferation, differentiation, and migration (Chen et al. , 2018a; Chen et al. , 2018b; Wang et al. , 2019a; Wang et al. , 2019b). Moreover, miR expression is highly related to vascular remodeling and angiogenesis (Henn et al. , 2019). Cai et al. found that miR-24 attenuated VR under diabetic conditions (Cai et al. , 2019). Downregulation of miR-224 aggravated VR in acute coronary syndrome (Xu et al. , 2019). MiR-1 overexpression mitigated pulmonary VR, thereby protecting against the progression of pulmonary hypertension (Sysol et al. , 2018).

MiR-26a, a highly conserved post-transcriptional regulator, can inhibit the proliferation and migration of VSMCs after vascular injury (Tanet al. , 2017) and protect VSMCs against oxidative stress (Penget al. , 2018). Thus, miR-26a may be a therapeutic target for vascular dysfunction. Moreover, the serum level of mir-26a was found altered in patients with hypertension (Yang et al. , 2018). However, the role and molecular mechanisms of miR-26a in hypertensive VR need to be clarified.

Our research aimed to elucidate the role of miR-26a in hypertensive VR and to reveal the potential miRdependent mechanisms. We present evidence that miR-26a plays a protective role in hypertensive VR. AngII-activated Smad3 inhibited miR-26a expression, which in turn promoted Smad3 activation by targeting Smad4, thereby forming a Smads/miR-26a positive feedback loop and further downregulating miR-26a. Downregulation of miR-26 further led to VR by promoting connective tissue growth factor (CTGF) expression and the enhancer of zeste homolog 2 (EZH2) /p21 pathway.

\section{Materials and Methods}

\subsection{Animals and experimental protocols}


Seven-week-old male spontaneously hypertensive rats (SHRs; $190 \pm 10 \mathrm{~g}$ ) and Wistar Kyoto rats (WKYs; $200 \pm 10 \mathrm{~g}$ ) were purchased from Beijing Wei Tong Lihua Experimental Animal Center. Ten-week-old male C57BL/6 mice $(25 \pm 2 \mathrm{~g})$ were purchased from the Laboratory Animal Center of Xi'an Jiaotong University. Animals were housed in a room with a controlled environment $\left(12 / 12\right.$-h light/dark cycle, $22-25^{\circ} \mathrm{C}, 50-60 \%$ relative humidity) with free access to normal diet and water. After study, all animals were anesthetized by inhalation of $1-2.5 \%$ isoflurane, and then euthanized by cervical dislocation. All animal experimental protocols were approved by the local and national ethical committee and conformed to NIH guidelines and the recommendations made by the British Journal of Pharmacology.

After 1 week of adaptation, SHRs were randomly divided into 3 groups of 8 rats each: recombinant adenoassociated virus (rAAV)-miR-26a (150 $\mu$ l, i.v.); rAAV-GFP $(150 \mu$ l, i.v.) (a total dose of viral particles was $2 \times 10^{11}$ v.g.; the rAAV vectors we used were rAAV of type-9; rAAV of miR-26a and vector controls was synthesized by Han Heng Biotechnology, China); and SHR-Ctrl (normal saline $150 \mu l$, i.v.). Six WKY rats were normal controls (normal saline $150 \mu$ l, i.v.). Systolic blood pressure (SBP) of the caudal artery of rats was measured every 3 to 5 days by using a non-invasive BP analysis system (BP-2000 SERIESII, Visitech, USA). After 3 weeks, plasma and thoracic aortas of rats were collected.

For mice experiments, C57BL $/ 6$ mice ( $\mathrm{n}=6$ per group) received AngII (Merck, USA, $2.0 \mathrm{mg} / \mathrm{kg} / \mathrm{d}$ ) or normal saline subcutaneously by implanted ALZETR 2002 minipumps under isoflurane anesthesia. Two weeks later, plasma and thoracic aortas of mice were collected.

\subsection{Histology}

Thoracic aorta sections were fixed in $4 \%$ paraformaldehyde, then embedded in paraffin. Morphological characteristics of thoracic aorta were assessed by hematoxylin-eosin (H\&E) staining. The media thickness (MT) of thoracic aorta was defined as the region between the internal and external elastic laminae. The MT/lumen diameter (LD) represented the level of VR. Thoracic aorta fibrosis was assessed by Masson's trichrome staining. Collagen volume fraction $(\mathrm{CVF})$ was quantified by measuring the ratio of blue fibrotic area to total thoracic aorta area.

\subsection{Immunohistochemical staining}

Aortic tissue sections $(4 \mu \mathrm{m})$ were deparaffinized, and endogenous peroxidase activity was quenched with $\mathrm{H}_{2} \mathrm{O}_{2}$. The sections were incubated with anti- $\alpha$-actin (dilution 1:200) and anti-proliferating cell nuclear antigen (PCNA; dilution 1:100) antibody at $4^{\circ} \mathrm{C}$ overnight, then with secondary antibody. After staining, each section was analyzed by confocal microscopy (DS-Fi1-Eclipse, Nikon).

\subsection{Cell culture}

VSMCs were prepared from thoracic aorta of 50-day-old female Sprague-Dawley rats. Aortic VSMCs were cultured in Dulbecco's modified Eagle's medium (Gibco, USA) with $10 \%$ fetal bovine serum (BI, USA) and $1 \%$ penicillin/streptomycin at $37 \mathrm{deg} \mathrm{C}$ in a $5 \% \mathrm{CO}_{2}$ humidified incubator. Immunohistochemical staining revealed positivity for smooth muscle $\alpha$-actin (Wanleibio, China) and negativity for CD105 (Wanleibio, China), indicating that primary VSMCs of rats were successfully isolated. Cells in passages 3-9 were used for all experiments.

\subsection{Transfection}

After 24-h incubation in serum-free medium, VSMCs were transfected with the miR-26a mimic (JTS Scientific, China) or miR-26a inhibitor (JTS Scientific, China) or a corresponding negative control (NC) or siRNA (Wanleibio, China) or SB431542 (MCE, China) according to the manufacturer's instructions. After 24- or 48-h transfection, cells were treated with AngII $\left(10^{-7} \mathrm{~mol} / \mathrm{L}\right.$ for $\left.24 \mathrm{~h}\right)$.

\subsection{CCK-8 assay}

VSMCs were cultivated in 96-well plates, with $4 \times 10^{3}$ cells in each well, and 5 duplicate wells were designed for each group. A 10- $\mu \mathrm{L}$ amount of CCK-8 solution (Wanleibio, China) was added after $24 \mathrm{~h}$ and incubated 
for $1 \mathrm{~h}$ under $5 \% \mathrm{CO}_{2}$ at 37 . A microplate reader (BioTek, USA) was used to detect optical density (OD) values at $450 \mathrm{~nm}$, which represents cell proliferation.

\subsection{Cell cycle assay}

Each group of VSMCs was washed twice with ice-cold phosphate buffered saline (PBS); cells were harvested by centrifugation and re-suspended in PBS, then fixed and permeabilized with $70 \%$ ethanol. Cells were washed twice in ice-cold PBS to remove ethanol, then treated with $500 \mu \mathrm{l}$ propidium iodide (PI; Wanleibio, China) in the dark at $4^{\circ} \mathrm{C}$ for $30 \mathrm{~min}$. Flow cytometry (ACEABio, NovoCyte, USA) was used to detect cell cycle.

\subsection{Immunofluorescence cell staining}

Cultured VSMCs were washed three times with PBS and fixed with $4 \%$ paraformaldehyde for $15 \mathrm{~min}$, followed by permeabilization with $0.1 \%$ Triton-X-100 (Beyotime, China) for $30 \mathrm{~min}$. Then cells were washed three times with PBS and incubated with anti-phospho-Smad3 (anti-p-Smad3) antibody (1:200 dilutions, ab52903, Abcam, England) overnight at $4^{\circ} \mathrm{C}$, followed by secondary goat anti-rabbit IgG-Cy3 antibody (1:200 dilutions, A0516, Beyotime, China) incubated for $1 \mathrm{~h}$ at room temperature. Nuclear staining of the cells involved using 4'6-diamidino-2-phenylindole dihydrochloride (DAPI, Beyotime, China) for 5 min at room temperature. Images were captured by fluorescence microscopy (OLUMPUS, Japan).

\subsection{ELISA}

Levels of collagen I and III (Col I and III) were detected by using ELISA kits (Cloud Clone Corp., China) according to the manufacturer's recommendations, and the absorbance at $450 \mathrm{~nm}$ was read. The relative Col I and III levels were calculated according to the standard concentration-absorbency curve.

\subsection{Quantitative real-time PCR (qRT-PCR)}

Total RNA from plasma, tissues or cells was extracted with Trizol reagent (Thermo Fisher Scientific, USA). cDNA was synthesized by using the miRNA Reverse Transcription System kit (TaKaRa, Japan) or $2 \times$ SYBR real-time PCR premixture (Bioteke Corp., China), following the manufacturer's protocol. Real-time PCR was performed with the StepOnePLus Real-Time PCR System. The primer sequences and conditions for amplification are in Supplemental Table S2 and S2, respectively. U6 or $\beta$-actin was the internal reference. The relative expression of mRNAs and miR was calculated by the $2^{-\mathrm{t}}$ method.

\subsection{Western blot analysis}

Protein was extracted from tissues or cells by using RIPA lysate (Hat Biotechnology, China). After quantification with bicinchoninic acid, equal amounts of protein were loaded and separated by SDS-PAGE, then transferred electrophoretically to polyvinylidene difluoride membranes, which were incubated with primary antibodies overnight at 4, followed by a 1:5000 dilution of goat anti-rabbit IgG antibody (EK020, Zhuangzhi Biotech, China) at room temperature. Primary antibodies were for CTGF (1:1000, ab6992), Smad3 (1:1000, ab40854), Smad4 (1:1000, ab40759), p-Smad3 (1:500, ab193297), cyclin D2 (1:1000, ab230883), p21 (1:500, ab109199; all Abcam, England); EZH2 (1:500, 5246; CST, USA); Col I (1:500, WL0088), Col III (1:500, WL0318), $\beta$-actin (1:500, WL01372; all Wanleibio, China), and glyceraldehyde-3-phosphate dehydrogenase (GAPDH; 1:1000, AF7021, Affinity Biosciences, USA). Protein bands were visualized by using a potent ECL kit (KF001, Affinity Biosciences, USA) and the Gene Company imaging system (China). GAPDH or $\beta$-actin was an internal reference.

\subsection{Chromatin immunoprecipitation (ChIP) assay}

$1 \%$ formaldehyde was used to cross-link VSMCs for $10 \mathrm{~min}$ at room temperature, then glycine $(0.125 \mathrm{M})$ was added for 5 min. The ChIP assay was performed with Smd3 or lgG antibody (Abcam, USA) and the ChIP kit (Wanleibio, China) according to the manufacturer's instructions. Bound DNA fragments were subjected to PCR. For PCR, $2 \mu \mathrm{L}$ of DNA was amplified with $2 \times$ Taq PCR Master-mix (Bioteke Corp., China), using a primer pair specific to the binding region of the miR-26a promoter. The primer sequences and the condition 
are in Supplemental Table S1 and S2, respectively. Finally, PCR products were visualized on 2\% agarose gels.

\subsection{Luciferase reporter assay}

The mutant-type (MuT) 3'-UTR of EZH2, CTGF and Smad4 was constructed. The WT or MuT 3'-UTR of EZH2, CTGF and Smad4 were inserted into pMIRGLO vectors (Promega, USA). The miR-26a mimic or miR-negative control and WT or MuT 3'-UTR of EZH2, CTGF and Smad4 vector were co-transfected into HEK293T cells (Wanleibio, China). After $48 \mathrm{~h}$, cells were lysed, and luciferase activity was determined by using a luciferase assay kit (Promega, USA) and multi-function microplate reader (TECAN, M200Pro, Switzerland).

\subsection{Data and statistical analysis}

The data and statistical analysis comply with the recommendations of the British Journal of Pharmacology on experimental design and analysis in pharmacology (Curtis et al. , 2018). All data are expressed as mean +SD and GraphPad Prism 6 software was performed to statistical analysis. To determine differences between groups with one factor, data were tested using unpaired Student's t-test for two group's comparison, and oneway ANOVA with Tukey's post hoc test analysis for multiple group comparisons. To determine differences between groups with multiple factors, data were tested using two-way ANOVA followed by Bonferroni's test. Post hoc tests were run only when $\mathrm{F}$ achieved $\mathrm{P}<0.05$. Differences were considered statistically significant at $\mathrm{P}<0.05$. All measurements were undertaken only for $\mathrm{n}[?] 5$.

\section{Results}

\subsection{Ang II regulated miR-26a expression both in vivo and in vitro}

MiR-26a expression was reduced in AngII-treated VSMCs dose- and time-dependently as compared with the control (Fig. 1a, b), so AngII negatively regulated the expression of miR-26a. In vivo, the relative level of miR-26a was lower in the thoracic aorta and plasma of AngII-treated mice than the control (Fig. 1c), which indicated Ang II regulated miR-26a expression both in vivo and in vitro.

\section{2 miR-26a had a protective effect on hypertensive VR}

To evaluate the role of miR-26a in hypertensive VR, rAAV vectors were constructed to overexpress miR26a in SHRs. As expected, rAAV-miR-26a transfection significantly increased the expression of miR-26a in aortas and plasma of SHRs (Fig. 2a). Meanwhile, rAAV-miR-26a prevented systolic blood pressure (SBP) from continuously increasing in SHRs (Supplemental Fig. S1). To evaluate VR, thoracic aortas underwent H\&E staining. As compared with normal WKYs, in SHRs, the media of the thoracic aorta was significantly thickened. However, miR-26a overexpression could reduce the medial thickening of the thoracic aorta caused by hypertension. The MT/LD ratio declined profoundly with miR-26a overexpression (Fig. 2b, c; rAAVmiR-26a vs rAAV-GFP and SHR-Ctrl was 5.430 +- 0.2943 vs $6.793+-0.4130$ and 7.575 +- $1.584 ; \mathrm{P}<0.05)$. Immunohistochemistry for smooth muscle $\alpha$-actin showed well-distributed smooth muscle and intact intima in WKY rats. However, VSMCs formed intima hyperplasia and parts of the vascular walls protruded into the aortic lumen in the SHR-Ctrl and rAAV-GFP groups. rAAV-miR-26a treatment significantly improved smooth muscle alignment and integrity (Fig. 2b).

\section{3 miR-26a ameliorates extracellular matrix (ECM) production in vivo and in vitro.}

ECM is mainly composed of collagen, so we observed and quantified CVF in vivo by Masson's trichrome staining. Trichrome staining and CVF were reduced in SHRs transfected with miR-26a as compared with rAAV-GFP and SHR-Ctrl groups (Fig. 3a, b; $31.26 \pm 3.902$ vs $54.25 \pm 5.987$ and $56.17 \pm 7.218, \mathrm{P}<0.05$ ). Then, we transfected miR-26a mimic and inhibitor into AngII-induced VSMCs to evaluate the effect of miR26 a on collagen production in vitro. On qRT-PCR, miR-26a was significantly elevated by miR-26a mimic treatment and markedly inhibited by miR-26a inhibitor treatment in AngII-treated VSMCs; neither of their negative controls (NCs) promoted or suppressed miR-26a expression (Fig. 3c). As expected, miR-26a mimic overexpression significantly suppressed and miR-26a inhibition enhanced the mRNA and protein expression 
of Col I and Col III according to ELISA, qRT-PCR and western blot analysis (Fig. 3d-f). In short, miR-26a inhibited collagen deposition both in vivo and in vitro.

\section{4 miR-26a inhibits VSMC proliferation in vivo and in vitro.}

The development of hypertensive VR is associated with the excessive proliferation of VSMCs. To determine whether miR-26a affected VSMC proliferation, immunohistochemistry for PCNA was used to test VSMC proliferation in thoracic aortas of SHRs and WKY rats. Cells with brown staining were defined as PCNApositive cells. The proportion of PCNA-positive cells (ratio of number of PCNA-positive cells to total number of sampled cells) was lower with rAAV-miR-26a overexpression than in vector- and vehicle-treated controls (Fig. 4a, b; $0.08801 \pm 0.01623$ vs $0.4440 \pm 0.04262$ and $0.4496 \pm 0.01413, \mathrm{P}<0.05$ ). We used CCK-8 assay and flow cytometry to measure the proliferation of VSMCs in vitro. Upregulation of miR-26a reduced the proliferation of AngII-induced VSMCs and retained cells in the G1 phase (Fig. 4c-e). miR-26a inhibitor treatment had the opposite effect, promoting cell proliferation.

\section{5 miR-26a suppresses ECM deposition by directly targeting CTGF.}

CTGF is essential for the synthesis of collagen (Liang et al. ,2014). We hypothesized that miR-26a attenuates ECM accumulation by regulating CTGF. In vivo data shown in Figure 5a support this hypothesis: CTGF protein level was significantly downregulated in SHR thoracic aortas with rAAV-miR-26a overexpression. The effect of miR-26a was further confirmed in vitro: miR-26a mimic suppressed CTGF expression in AngII-induced VSMCs and miR-26a inhibitor increased CTGF protein and mRNA levels in vitro (Fig. 5b, c). Bioinformatics analysis by using Targetscan (www.targetscan.org) predicted CTGF as a potential target gene of miR-26a (Fig. 5d). By using a reporter construct with the putative 3'-UTR miR-26a binding site of CTGF downstream of the luciferase gene, we found that miR-26a reduced luciferase activity, which provides experimental validation of CTGF as a target for miR-26a. Accordingly, after mutation of the binding site, the activity of luciferase was not altered (Fig. 5e), which confirms that the binding of miR-26a to the 3'-UTR site was necessary for silencing CTGF

\subsection{EZH2/p21 pathway mediates miR-26a dysregulation-induced VSMC proliferation.}

To explore the mechanisms of miR-26a-inhibited proliferation of VSMCs, we investigated the regulation of miR-26a of proliferation-related genes. Considering that rAAV-miR-26a overexpression decreased EZH2 protein level in the thoracic aorta of SHRs (Fig. 6a), we assumed that miR-26a inhibiting VSMC proliferation is mediated by EZH2. Consistent with in vivo findings, in AngII-induced VSMCs, the expression of EZH2 at both the protein and mRNA levels was significantly decreased after transfection with miR-26a mimic (Fig. 6b, e). Furthermore, Targetscan revealed that the miR-26a binding site within the 3'UTR of EZH2 mRNA is highly conserved (Fig. 6f). We further performed luciferase reporter assay and found reduced luciferase activity with miR-26a mimic transfection. However, miR-26a did not affect activity of the binding site mutation, which confirms that EZH2 is a target of miR-26a (Fig. 6g).

p21 belongs to a cell-cycle regulator that contributes to cell growth inhibition, and the expression of p21 is indirectly inhibited by EZH2 ( $\mathrm{Lu}$ et al. , 2011; Li et al. , 2019). In our study, p21 expression was negatively related to the expression of EZH2 both in vitro and in vivo (Fig. 6a, c), which suggests that miR-26a upregulated p21 level by directly suppressing EZH2 expression. In other words, miR-26a inhibited VSMC proliferation by regulating the EZH2/p21 pathway.

miR-26a can also regulate cell proliferation by directly targeting cyclin D2 (Zhou et al. , 2016). To explore whether miR-26a regulated cyclin D2 expression in VSMCs, we transfected the miR-26a mimic and inhibitor in AngII-induced VSMCs. The expression of cyclin D2 was markedly upregulated in AngII-induced VSMCs on transfection with miR-26a inhibitor and was reversed by transfection with the miR-26a mimic (Fig. 6d). Thus, miR-26a mitigates excessive VSMC proliferation by targeting the EZH2/p21 pathway and cyclin D2.

\subsection{AngII regulates miR-26a expression via Smad3 activation.}

PROMO analysis revealed three potential Smad3 binding sites in the miR-26a promoter (Fig. 7a). ChIP 
assay with VSMC lysates and anti-Smad3 antibody validated that Smad3 directly binds to the promoter of miR-26a to suppress its expression. Additionally, Smad3 exhibited stronger binding to the miR-26a promoter in AngII-induced VSMCs as compared with controls (Fig. 7b, c). Thus, AngII promoted Smad3 binding to the miR-26a promoter, thereby inhibiting miR-26a transcription. To further elaborate whether miR-26a downregulation was mediated by Smad3 in VSMCs, we used loss-of-function studies. We first constructed three siRNAs fragments of Smad3 to transfect VSMCs. Both qRT-PCR and western blot analysis confirmed that siRNA-3 silenced Smad3 the best (Supplemental Fig. S2a, b). Next, we used siRNA or SB431542, which inhibited Smad3 expression or activation, respectively, and observed increased miR-26a expression in AngII-induced VSMCs (Fig. 7d-f), which indicates that Smad3 negatively regulates miR-26a expression. These results indicate that AngII regulates miR-26a expression via Smad3 activation.

\section{8 miR-26a inversely regulates the activation of Smad3}

We transfected AngII-induced VSMCs with an miR-26a mimic, which showed high-transfection efficiency (Fig. 8a). Also, transfection of an miR-26a mimic significantly decreased p-Smad3 protein expression (Fig. 8b). Additionally, upregulation of miR-26a inhibited nuclear translocation of p-Smad3 (Fig. 8c). These results implied that miR-26a can also inversely inhibit Smad3 activation.

\section{9 miR-26a inhibits Smad3 activation by targeting Smad4}

We next sought to determine how miR-26a regulates Smad3 activation. MiRanda (www.microRNA.org) analysis indicated that Smad4 contains a binding site for miR-26a (Fig. 9a). We confirmed that Smad4 was a direct target of miR-26a by luciferase reporter assay (Fig. 9b). Consistently, Smad4 expression was significantly downregulated with miR-26a mimic overexpression, whereas miR-26a inhibitor treatment increased the protein level of Smad4 in AngII-induced VSMCs (Fig. 9c, d).

To explore the effect of Smad4 on Smad3 activation, we examined siRNA-mediated knockdown of reduced level of Smad4 in VSMCs. We selected siRNA with the best silencing effect on Smad4 to transfect AngIItreated VSMCs (Supplemental Fig. S2c, d). Smad4 expression was highly relevant for the expression and nuclear translocation of p-Smad3. Inhibition of Smad4 could reduce p-Smad3 protein expression and decreased the nuclear translocation of p-Smad3 (Fig. 9e-h). Therefore, Smad4 promoted the activation of Smad3. In summary, miR-26a regulated Smad3 activation by targeting Smad4.

Combined with previous results (Fig. 7-9), a positive feedback loop between miR-26a and Smad3/4 may be involved in hypertensive VR (Fig. 10).

\section{Discussion}

In this study, we evaluated the role and mechanisms of miR-26a in controlling hypertensive VR (Fig. 10). MiR-26a was significantly downregulated in the thoracic aorta and plasma of SHRs with VR. Overexpression of miR-26a effectively reduced ECM deposition and VSMC hyperproliferation both in vivo and in vitro. Also, AngII potentiated Smad3 binding to the miR-26a promoter, inhibiting miR-26a transcription, which in turn promoted Smad3 activation by upregulating Smad4, thereby further downregulating miR-26a. These findings reveal a possible new positive feedback loop between miR-26a and Smad3/4, which may be related to hypertensive VR. Moreover, miR-26a mitigated ECM accumulation and excessive VSMC proliferation in AngII-induced VSMCs by targeting CTGF and the EZH2/p21 pathway, respectively. Our study suggests that miR-26a is a protective molecule in hypertensive VR and can be considered a promising target for treating hypertensive VR.

The crucial finding of this study is that miR-26a plays a protective role in hypertensive VR. Previous studies have shown that miR-26a is closely related to cardiovascular diseases. Li et al. found low expression of miR26a in plasma of patients with acute myocardial infarction as compared with healthy people (Li et al. , 2015). Zhang et al. showed that miR-26a plays a role in the process of myocardial fibrosis after acute myocardial infarction by inhibiting phosphatase and tensin homolog expression, enhancing matrix metalloproteinase 9 level and promoting the PI3K/AKT pathway (Zhanget al. , 2018). Chiang et al. discovered that miR-26a ameliorates cardiac dysfunction and fibrosis in myocardial infarction (Chiang et al. , 2020). In this study, we 
first observed that miR-26a expression was downregulated in the thoracic aorta and plasma of SHRs. We next used rAAV-miR-26a to overexpress miR-26a in the thoracic aorta and plasma of SHRs. The overexpression of miR-26a reduced media thickening of the thoracic aorta and diminished the severity of VR induced by hypertension. However, due to the limitations of this experiment, we have not been able to obtain data on the extent of SHR aortic remodeling before miR-26a treatment. Therefore, our conclusion was based on the hypothesis that there was no difference in VR of SHRs in each group before intervention. Meanwhile, we found that overexpression of miR-26a appears to have an effect on blood pressure, it is unknown whether miR-26a directly reduces VR or if it is secondary to reduced blood pressure, which can continue to study in future.

One of the characteristics of VR is pathological remodeling of ECM, which is often accompanied by increased ECM release and collagen deposition (Ricard-Blum et al. ,2019). ECM is a structural scaffold of the blood vessel wall, which controls cellular functions in the pathological environment. When stimulated or injured, the deposition of ECM protein, especially Col I, alters the collagen/elastin ratio and hemodynamics, leading to increased vascular stiffness and affecting blood vessel function (Lee et al. , 2015). We found the CVF of the thoracic-aorta media layer reduced in miR-26a-overexpressed SHRs. In vitro studies showed that miR-26a mimic treatment inhibited but miR-26a inhibitor treatment promoted Col I and III expression in AngII-induced VSMCs. CTGF belongs to matricellular protein expressed by various cells in response to stimuli and is an important molecule prominently implicated in increased deposition of ECM (Li et al. , 2016; Petrosino et al. , 2019). Our results showed that that CTGF is a downstream target gene of miR-26a and the level of miR-26a was inversely associated with CTGF expression both in vivo and in vitro, so miR26a may diminish ECM deposition by targeting CTGF. Additionally, the Col I is a downstream target gene of miR-26a (Wei et al. , 2013). MiR-26a may directly inhibit collagen synthesis to reduce ECM deposition, which needs further study.

Another pathological manifestation of hypertensive VR is excessive VSMC proliferation, which is determined by the cell cycle (Deniset et al. , 2018; Lu et al. , 2018; He et al. , 2019). EZH2 accelerates the cell cycle, whereas p21 arrests the cell cycle (Karimianet al. , 2016; Dai et al. , 2018). EZH2/p21 has been found a critical signaling pathway to regulate cell proliferation (Lu et al. , 2011; Li et al. , 2019). We found that transfection with rAAV-miR-26a suppressed PCNA and EZH2 expression, but p21 was upregulated in the thoracic aorta of SHRs. Similarly, in vitro experiments showed that miR-26a inhibited the progression of the cell cycle accompanied by downregulated protein level of EZH2, whereas p21 level was correspondingly increased in AngII-induced VSMCs; miR-26a inhibitor treatment had the opposite result. Furthermore, EZH2 is a target gene of miR-26a, so miR-26a participates in VSMC proliferation by targeting the EZH2/p21 pathway. Cyclin D2 can promote the G1/S transition (Gul et al. , 2018; Pei et al. , 2018), and previous studies with luciferase reporter assay showed that cyclin D2 is also a target gene of miR-26a (Zhou et al. , 2016). Consistently, we found that miR-26a suppressed cyclin D2 expression in AngII-induced VSMCs. Thus, miR-26a may be involved in excessive VSMC proliferation by directly regulating cyclin D2 apart from targeting the EZH2/p21 pathway.

Our study confirmed that AngII regulates miR-26a level dose- and time-dependently. However, how AngII regulates miR-26a expression is unclear. Some studies demonstrated that miRs are subject to sophisticated regulation, and superposition of subtle changes in the expression of several molecules may be responsible for miRs exerting their effects (Krol et al. , 2010; Liang et al. , 2014). Previous studies determined that miR-26a inhibits TGF $\beta$-dependent Smad signaling (Leeper et al. , 2011). We focused on TGFß-independent Smad signal regulatory mechanisms of miR-26a. In this study, we validated that Smad3 could directly suppress miR-26a expression, and AngII could promote this effect; AngII regulates miR-26a by activating Smad3. Previous studies reported a functional crosstalk between miR-26a and other molecules via a feedback loop (Jiang et al. , 2018; Liuet al. , 2018). We found that miR-26a downregulation enhanced Smad4 expression via targeted regulation and then induced Smad3 activation and p-Smad3 nuclear translocation. Smad3 activation caused further downregulation of miR-26a, thereby forming a Smads/miR-26a positive feedback loop. Once the loop is activated, it will run repeatedly, eventually leading to increased ECM accumulation, excessive VSMC proliferation and VR. 
To sum up, miR-26a plays a protective role in hypertensive VR. The effect of miR-26a on VR was mediated by activation of a Smads/miR-26a positive feedback loop. In addition, miR-26a inhibited ECM deposition by targeting CTGF and attenuated VSMC proliferation by regulating the EZH2/p21 pathway. We clarify the functions and mechanisms of miR-26a in hypertensive VR and suggest that miR-26a may be a novel therapeutic target of hypertensive VR.

\section{Acknowledgements}

The authors thank the Research and Experiment Center of the Second Affiliated Hospital of Xi'an Jiaotong University and the Experimental Animal Center of the Medical Department of Xi'an Jiaotong University for technical assistance and experimental equipments. This work was supported by the National Natural Science Foundation of China (81570382, 81872563).

\section{Conflict of interest}

The authors declare that they have no competing interests.

\section{Author contributions}

D.G. conceived and designed this study. W.Z., Q.Z., X.X., L.Y., M.X., C.C., R.W. and W.C. conducted the experiments and analysed the data. D.G. and W.Z. drafted the manuscript and coordinated manuscript revisions. Prof. X. N. provided suggestion for the experiments. The manuscript has been reviewed and approved by all authors.

\section{Declaration of transparency and scientific rigour}

This Declaration acknowledges that this paper adheres to the principles for transparent reporting and scientific rigour of preclinical research as stated in the BJP guidelines for Design \& Analysis, Immunoblotting and Immunochemistry, and Animal Experimentation, and as recommended by funding agencies, publishers and other organisations engaged with supporting research.

\section{References}

Brown IAM, Diederich L, Good ME, DeLalio LJ, Murphy SA, Cortese-Krott MM, et al. (2018). Vascular Smooth Muscle Remodeling in Conductive and Resistance Arteries in Hypertension.Arteriosclerosis, thrombosis, and vascular biology 38:1969-1985.

Cai W, Zhang J, Yang J, Fan Z, Liu X, Gao W, et al. (2019). MicroRNA-24 attenuates vascular remodeling in diabetic rats through PI3K/Akt signaling pathway. Nutrition, metabolism, and cardiovascular diseases : NMCD 29: 621-632.

Chen WJ, Chen YH, Hsu YJ, Lin KH, Yeh YH (2018a). MicroRNA-132 targeting PTEN contributes to cilostazol-promoted vascular smooth muscle cell differentiation. Atherosclerosis 274: 1-7.

Chen Z, Wang M, Huang K, He Q, Li H, Chang G (2018b). MicroRNA-125b Affects Vascular Smooth Muscle Cell Function by Targeting Serum Response Factor. Cellular physiology and biochemistry : international journal of experimental cellular physiology, biochemistry, and pharmacology 46: 1566-1580.

Chiang MH, Liang CJ, Lin LC, Yang YF, Huang CC, Chen YH, et al.(2020). miR-26a attenuates cardiac apoptosis and fibrosis by targeting ataxia-telangiectasia mutated in myocardial infarction. Journal of cellular physiology .

Curtis MJ, Alexander S, Cirino G, Docherty JR, George CH, Giembycz MA, et al. (2018). Experimental design and analysis and their reporting II: updated and simplified guidance for authors and peer reviewers. British journal of pharmacology 175: 987-993.

Dai X, Chen C, Yang Q, Xue J, Chen X, Sun B, et al. (2018). Exosomal circRNA_100284 from arsenitetransformed cells, via microRNA-217 regulation of EZH2, is involved in the malignant transformation of 
human hepatic cells by accelerating the cell cycle and promoting cell proliferation. Cell death $\&$ disease $9: 454$.

Das S, Zhang E, Senapati P, Amaram V, Reddy MA, Stapleton K, et al. (2018). A Novel Angiotensin IIInduced Long Noncoding RNA Giver Regulates Oxidative Stress, Inflammation, and Proliferation in Vascular Smooth Muscle Cells. Circulation research 123:1298-1312.

Deniset JF, Hedley TE, Hlavackova M, Chahine MN, Dibrov E, O'Hara K, et al. (2018). Heat shock protein 60 involvement in vascular smooth muscle cell proliferation. Cellular signalling47: 44-51.

Gul A, Leyland-Jones B, Dey N, De P (2018). A combination of the PI3K pathway inhibitor plus cell cycle pathway inhibitor to combat endocrine resistance in hormone receptor-positive breast cancer: a genomic algorithm-based treatment approach. American journal of cancer research 8: 2359-2376.

Hayashi K, Naiki T (2009). Adaptation and remodeling of vascular wall; biomechanical response to hypertension. Journal of the mechanical behavior of biomedical materials 2: 3-19.

He F, Chu JF, Chen HW, Lin W, Lin S, Chen YQ, et al. (2019). Qingxuan Jiangya Decoction () Prevents Blood Pressure Elevation and Ameliorates Vascular Structural Remodeling via Modulating TGF- beta 1/Smad Pathway in Spontaneously Hypertensive Rats. Chinese journal of integrative medicine .

Henn D, Abu-Halima M, Wermke D, Falkner F, Thomas B, Kopple C, et al. (2019). MicroRNA-regulated pathways of flow-stimulated angiogenesis and vascular remodeling in vivo. Journal of translational medicine 17: 22 .

Jiang H, Chen Y, Yu T, Zhao X, Shan H, Sun J, et al. (2018). Inhibition of lncRNA PFRL prevents pulmonary fibrosis by disrupting the miR-26a/smad2 loop. American journal of physiology. Lung cellular and molecular physiology 315: L563-1575.

Karimian A, Ahmadi Y, Yousefi B (2016). Multiple functions of p21 in cell cycle, apoptosis and transcriptional regulation after DNA damage.DNA repair 42: 63-71.

Krol J, Loedige I, Filipowicz W (2010). The widespread regulation of microRNA biogenesis, function and decay. Nature reviews. Genetics11: 597-610.

Lee TH, Sottile J, Chiang HY (2015). Collagen inhibitory peptide R1R2 mediates vascular remodeling by decreasing inflammation and smooth muscle cell activation. PloS one 10: e0117356.

Leeper NJ, Raiesdana A, Kojima Y, Chun HJ, Azuma J, Maegdefessel L, et al. (2011). MicroRNA-26a is a novel regulator of vascular smooth muscle cell function. Journal of cellular physiology226: 1035-1043.

Lemarie CA, Tharaux PL, Lehoux S (2010). Extracellular matrix alterations in hypertensive vascular remodeling. Journal of molecular and cellular cardiology 48: 433-439.

Li C, Chen X, Huang J, Sun Q, Wang L (2015). Clinical impact of circulating miR-26a, miR-191, and miR-208b in plasma of patients with acute myocardial infarction. European journal of medical research20: 58.

Li C, Zhen G, Chai Y, Xie L, Crane JL, Farber E, et al. (2016). RhoA determines lineage fate of mesenchymal stem cells by modulating CTGF-VEGF complex in extracellular matrix. Nature communications7: 11455 .

Li Z, Yu D, Li H, Lv Y, Li S (2019). Long noncoding RNA UCA1 confers tamoxifen resistance in breast cancer endocrinotherapy through regulation of the EZH2/p21 axis and the PI3K/AKT signaling pathway.International journal of oncology 54: 1033-1042.

Liang H, Xu C, Pan Z, Zhang Y, Xu Z, Chen Y, et al. (2014). The antifibrotic effects and mechanisms of microRNA-26a action in idiopathic pulmonary fibrosis. Molecular therapy: the journal of the American Society of Gene Therapy 22: 1122-1133. 
Liu J, Li X, Wang M, Xiao G, Yang G, Wang H, et al. (2018). A miR-26a/E2F7 feedback loop contributes to tamoxifen resistance in ER-positive breast cancer. International journal of oncology53: 1601-1612.

Lu J, He ML, Wang L, Chen Y, Liu X, Dong Q, et al. (2011). MiR-26a inhibits cell growth and tumorigenesis of nasopharyngeal carcinoma through repression of EZH2. Cancer research71: 225-233.

Lu QB, Wang HP, Tang ZH, Cheng H, Du Q, Wang YB, et al. (2018). Nesfatin-1 functions as a switch for phenotype transformation and proliferation of VSMCs in hypertensive vascular remodeling.Biochimica et biophysica acta. Molecular basis of disease1864: 2154-2168.

Magnussen CG (2017). Carotid artery intima-media thickness and hypertensive heart disease: a short review. Clinical hypertension23: 7 .

Mulvany MJ (2012). Small artery remodelling in hypertension. Basic \& clinical pharmacology $\&$ toxicology 110: 49-55.

Pei Y, Singh RK, Shukla SK, Lang F, Zhang S, Robertson ES (2018). Epstein-Barr Virus Nuclear Antigen 3C Facilitates Cell Proliferation by Regulating Cyclin D2. Journal of virology 92.

Peng J, He X, Zhang L, Liu P (2018). MicroRNA26a protects vascular smooth muscle cells against H2O2induced injury through activation of the PTEN/AKT/mTOR pathway. International journal of molecular medicine 42: 1367-1378.

Petrosino JM, Leask A, Accornero F (2019). Genetic manipulation of CCN2/CTGF unveils cell-specific ECM-remodeling effects in injured skeletal muscle. FASEB journal : official publication of the Federation of American Societies for Experimental Biology 33:2047-2057.

Ricard-Blum S, Vallet SD (2019). Fragments generated upon extracellular matrix remodeling: Biological regulators and potential drugs. Matrix biology : journal of the International Society for Matrix Biology 75-76: 170-189.

Sysol JR, Chen J, Singla S, Zhao S, Comhair S, Natarajan V, et al. (2018). Micro-RNA-1 is decreased by hypoxia and contributes to the development of pulmonary vascular remodeling via regulation of sphingosine kinase 1. American journal of physiology. Lung cellular and molecular physiology 314: L461-1472.

Tan J, Yang L, Liu C, Yan Z (2017). MicroRNA-26a targets MAPK6 to inhibit smooth muscle cell proliferation and vein graft neointimal hyperplasia. Scientific reports 7: 46602.

Wang D, Atanasov AG (2019a). The microRNAs Regulating Vascular Smooth Muscle Cell Proliferation: A Minireview. International journal of molecular sciences 20.

Wang J, Zhang C, Li C, Zhao D, Li S, Ma L, et al. (2019b). MicroRNA-92a promotes vascular smooth muscle cell proliferation and migration through the ROCK/MLCK signalling pathway. Journal of cellular and molecular medicine 23: 3696-3710.

Wei C, Kim IK, Kumar S, Jayasinghe S, Hong N, Castoldi G, et al.(2013). NF-kappaB mediated miR-26a regulation in cardiac fibrosis. Journal of cellular physiology 228: 1433-1442.

Xu HM, Sui FH, Sun MH, Guo GL (2019). Downregulated microRNA-224 aggravates vulnerable atherosclerotic plaques and vascular remodeling in acute coronary syndrome through activation of the TGF-beta/Smad pathway. Journal of cellular physiology 234: 2537-2551.

Yang X, Niu X, Xiao Y, Lin K, Chen X (2018). MiRNA expression profiles in healthy OSAHS and OSAHS with arterial hypertension: potential diagnostic and early warning markers. Respiratory research19: 194.

Zhang CJ, Shi YN, Liao DF, Du K, Qin L (2019). [Molecular mechanism of vascular remodeling in hypertension and Chinese medicine intervention]. Sheng li xue bao : [Acta physiologica Sinica] 71: 235-247. 
Zhang S, Cui R (2018). The targeted regulation of miR-26a on PTEN-PI3K/AKT signaling pathway in myocardial fibrosis after myocardial infarction. European review for medical and pharmacological sciences 22: $523-531$.

Zhou J, Ju WQ, Yuan XP, Zhu XF, Wang DP, He XS (2016). miR-26a regulates mouse hepatocyte proliferation via directly targeting the 3' untranslated region of CCND2 and CCNE2. Hepatobiliary 83 pancreatic diseases international : HBPD INT 15: 65-72.

\section{Hosted file}

Figure.docx available at https://authorea.com/users/347366/articles/473101-the-antagonisticeffects-and-mechanisms-of-microrna-26a-action-in-hypertensive-vascular-remodeling 